\title{
The Lack of Relationship Between DST Nonsuppression in the Dexamethasone Suppression Test and EEG Abnormalities
}

\author{
William Pitchot, Michel Hansenne, and Marc Ansseau \\ Psychiatric Unit, Centre Hospitalier Universitaire du Sart Tilman, B-4000 Liège, Belgium
}

Received December 17, 1990

\begin{abstract}
Summary. As a recent study suggested a relationship between cortisol escape following dexamethasone suppression test (DST) and electroencephalogram (EEG) abnormalities, we tried to replicate these findings in 52 major depressive inpatients. A total of 23 patients exhibited DST nonsuppression (44\%) and 18 patients had EEG abnormalities (35\%). No relationship existed between DST and EEG results.
\end{abstract}

Key words: DST - Electroencephalogram - Depressive illness

\section{Introduction}

In the diagnostical confirmation of major depressive disorders, the dexamethasone suppression test (DST) has been proposed as a biological marker of the endogenous subtype [Carroll et al. 1981]. However, the post-DST cortisol level has been correlated with many other factors and thus appears rather unspecific. Recently, Miller and Nelson (1987) showed a relationship between cortisol nonsuppression and electroencephalogram (EEG) abnormalities. They found that the post-DST cortisol concentrations were more strongly related to abnormal EEG findings than to the diagnosis of melancholia. The aim of the present study was to replicate these findings, and specifically to compare EEG disturbances in major depressive patients with their DST results.

\section{Methods}

We assessed a sample of 52 inpatients admitted to the Psychiatric Unit of the University Hospital of Liège, Belgium. All patients fulfilled DSM-III-R criteria for a major depressive disorder and

Offprint requests to: $\mathrm{M}$. Ansseau had a score on the 17-item Hamilton rating scale for depression of at least 18 [Hamilton 1960]. The sample comprised 18 male and 34 female patients with age ranging from 23 to 62 years (mean age $=$ 47.0, SD 10.6 years). EEG and DST were performed within the first 2 weeks of hospitalisation. All patients were free of medical illness and particularly of epileptic disorders, and of all medication including psychotropic drugs known to interfere with the DST [Carroll et al. 1981]. The protocol was approved by the Ethical Committee of the University of Liège Medical School and all patients gave their informed consent.

The DST was performed according to the simplified procedure described by Carroll et al. (1981). Each patient received $1 \mathrm{mg}$ of dexamethasone at $11.00 \mathrm{p} . \mathrm{m}$. and a blood sample was collected at 4.00 p.m. the next day. Concentrations of total cortisol were determined by radioimmunoassay (RIA), with intra- and inter-assay coefficients of variation of 4.3 and $8.3 \%$, respectively [Sulon et al. 1978]. We considered DST nonsuppression for all cortisol values higher than $5 \mu \mathrm{g} / \mathrm{dl}$ [Carroll et al. 1981].

The electrode montage was the ten-twenty system [Jasper 1958] with hyperventilation in the middle of the recording. The EEGs were scored by graduate neurologists independently of our study and with an interscorer correlation coefficient of 0.85 . EEG abnormalities consisted of diffuse or localized theta activity not related to sleep and abnormally distributed, with a tendency to be focal. All the recordings with beta rhythm were excluded to avoid medication effect. We also rejected EEGs in 4 patients with an abnormal neurological examination. Therefore, we had only two categories of EEG results: normal and abnormal.

The relationship between DST (suppression vs nonsuppression) and EEG (normal vs abnormal) was assessed by the chi square procedure.

\section{Results}

A total of 23 patients exhibited DST nonsuppression $(44 \%)$ and 18 patients had EEG abnormalities (35\%). No relationship existed between DST and EEG results. Patients with EEG abnormalities exhibited less frequent DST nonsuppression $(6 / 18=33 \%)$ than those with normal EEG $(17 / 34=50 \%)$, but this difference did not reach statistical significance (chi square $=1.32, d f=1, P=$ $0.25)$. 


\section{Discussion}

In our study, we did not find any correlation between DST and EEG abnormalities. Patients with cortisol escape after dexamethasone did not show more EEG dysfunction than those with normal suppression. Our results are in contrast to those of Miller and Nelson (1987) where patients with DST nonsuppression tended to have more frequent abnormal EEGs than patients with DST suppression.

Several differences in the methodological approach could explain these discrepancies. First, we measured total cortisol by RIA for all patients, while a fluorometric technique was used for almost all the patients by Miller and Nelson. Second, our DST procedure was the same for the 52 patients with a blood sample drawn at 4.00 p.m., while blood samples were collected at either 4 or $11 \mathrm{p} . \mathrm{m}$. in the previous study. Third, we used only two categories in assessing the EEG (normal-abnormal) with more precise criteria of EEG abnormalities. Finally, we also tried to avoid pharmacological artifacts by excluding EEGs with beta rhythms.
In conclusion, our study did not confirm any relationship between EEG abnormalities and DST results. These findings should, however, be confirmed in prospective studies.

\section{References}

Carroll BJ, Feinberg M, Greden JF, Tarika J, Albala AA, Haskett RF, James NM, Kronfol Z, Lohr N, Steiner M, De Vigne JP, Young E (1981) A specific laboratory test for the diagnosis of melancholia. Arch Gen Psychiatry 38:15-22

Hamilton M (1960) A rating scale for depression. J Neurol Neurosurg Psychiatry 23:56-62

Jasper HH (1958) The Ten-Twenty system of the International Federation. Electroencephalogr Clin Neurophysiol 10:371

Miller KS, Nelson JC (1987) Dexamethasone nonsuppression and EEG abnormalities. Biol Psychiatry 22:1151-1155

Sulon J, Demey-Ponsart E, Bauduin E, Sodoyez JC (1978) Radioimmunoassay of corticosterone, cortisone and cortisol. Their application to human cord and maternal plasma. J Steroid Biochem 9:671-676 